\title{
Factors affecting the clinical measurement of visuo-spatial neglect
}

\author{
L. Pizzamiglio', C. Bergego², P. Halligan ${ }^{3}$, V. Homberg ${ }^{4}$, I. Robertson ${ }^{5}$, \\ E. Weber ${ }^{6}$, B. Wilson ${ }^{5}$, P. Zoccolotti ${ }^{1}$ and G. Deloche ${ }^{2}$
}

'Univ. "La Sapienza" of Roma and Clinica S. Lucia, Italy, ${ }^{2}$ Hopital de la Salpetriere, Paris, France, ${ }^{3} \mathrm{MRC}$ Neuropsychology Unit and Rivermead Rehabilitation Centre, Oxford, UK, ${ }^{4}$ Neurologisches Therapiecentrum, Dusseldorf, Germany, ${ }^{5}$ MRC Applied Psychology Unit, Cambridge, UK, and ${ }^{6}$ Neurol. Reha-Zentrum, Bonn, Germany

Correspondence to: L. Pizzamiglio, Università di Roma "La Sapienza", Via dei Marsi, 78, 00185, Roma, Italy

\begin{abstract}
The present study examined a battery of tests to evaluate unilateral spatial neglect; the tests included different tasks involving several modalities of spatial exploration mapping perceptual, motor, attentional and personal or extrapersonal space dimensions. The subjects, 121 right-brain-damaged patients with unilateral neglect, were studied in seven laboratories in four European countries. Relationships among the various tests were examined by correlations, a cluster analysis and by an analysis of individual cases. Different sensitivity was found among various tests for detecting neglect performances. Both the cluster analysis and the single case analysis clearly showed a segregation between personal and extrapersonal neglect. Analysis of the large cluster, including a variety of tests of extrapersonal neglect, together with the study of single cases, suggests the possibility of differentiating the various manifestations of spatial neglect which can be interpreted on the basis of the descriptions of other individual cases previously reported in the literature. Finally, the present study indicated the relative stability of neglect following the acute phase and its independence from age.
\end{abstract}

Keywords: Assessment - Personal space - Visuo-spatial neglect

\section{INTRODUCTION}

The term "visual neglect" is used to indicate a variety of impaired behaviours which have in common the inability to attend to or represent one side of the space (see reviews in Jeannerod, 1987; Bisiach and Vallar, 1988; Robertson and Marshall, 1992). This condition is considerably more frequent (and severe) after damage to the right than to the left hemisphere (De Renzi, 1982). For the sake of presentation, we will provide examples which always refer to patients with right brain damage (and left-sided neglect).

In the very early stages after the onset of disease, the defect can be very evident under most circumstances and creates little diagnostic problem. The patient may respond to or detect only stimuli placed on the right. Furthermore, in a sizeable proportion of cases, left neglect may be accompanied by tonic deviation of the head and/or the eyes toward the side ipsilateral to the lesion (i.e. toward the right) (De Renzi et al., 1982).

Approximately 1 month after the CVA, the most conspicuous forms of neglect are relatively infrequent (Gainotti, 1968) and diagnosis is commonly based on a variety of standard tests. The most common of these involve tasks in which the patient has to cross out a number of segments, letters or symbols (cancellation tests), read words, sentences or short stories (reading tests), draw a figure from a model or from memory (drawing tests) or divide a line into two identical parts (bisection test). Asymmetrical pointing performance or misidentifications of the left part of the stimulus material are taken as an indication of left-sided neglect.

In general, performance in different tests used to measure neglect is positively correlated, apparently pointing to the relative ease of diagnosis of this defect. However, considerable individual variability from test to test is also reported (e.g. Horner et al., 1989; Halligan et al., 1989). As a result of this, estimates of its incidence can vary greatly ranging from $12 \%$ (Smith et al., 1983) to $85 \%$ (Hier et al., 1983). Part of this variability may be accounted for by differences in the psychometric characteristics of the tests as well as factors such as aetiology, time from onset of disease, and the size of lesion examined (Halligan et al., 1991 a). However, several recent reports in the literature indicate that neglect may fractionate in a 
number of different ways, indicating that several independent mechanisms may contribute to the clinical appearance of a severe form of neglect.

One basic distinction concerns the failure to process visual input in the space contralateral to the lesion and the difficulty of initiating and carrying out a motor response in the neglected space (Heilman et al., 1985). Tegner and Levander (1991), using a cancellation test viewed through a $90^{\circ}$ angle mirror which prevented a direct view of the stimulus, were able to separate the two forms of neglect. Patients failed either in responding to contralateral perceptual information (perceptual neglect) or in performing motor responses in the contralesional space (premotor neglect).

A second important distinction can be drawn on the basis of differences in the spatial properties of the stimulus. For example, a disassociation between neglect for extrapersonal and personal space has been documented in monkeys (e.g. Rizzolatti and Camarda, 1987). Furthermore, clinical evidence points to the need to conceptualize personal space as different from extrapersonal space (Brain, 1941; Bisiach et al., 1986; Guariglia and Antonucci, 1992). For example, Bisiach et al. (1986) noted that some patients with a right hemispheric lesion were unable or slow in searching for their left hand; however, a number of them were unimpaired in a standard cancellation test, pointing to the independence of the underlying deficits. A further differentiation has been proposed between near (within hand reach) and far (outside hand reach) extrapersonal space. Recently, Halligan and Marshall (1991) presented a patient who showed only neglect when bisecting a line placed in near but not far extrapersonal space. This disassociation may be relatively infrequent in humans (Pizzamiglio et al., 1989). However, it seems useful to note that most, if not all, standard tests of unilateral neglect consider only stimulus material placed in the space within hand reach of the patient.

Several observations indicate that deficits in basic attentional processes may contribute to determining performance on standard neglect tests. Using a cancellation task, Rapcsak et al. (1989) noted that there were more omissions on the left side if targets were interspersed with difficult-to-discriminate foils; performance progressively improved in the case of easier distractors and in the condition in which only targets were used. Rapcsak et al. (1989) posited that unilateral neglect can be worsened if the task poses a specific load on selective attention. Consistent with an attentional interpretation in a different study, it has also been found that the percentage of targets crossed out on a cancellation test decreased linearly with increasing number of distractors (Kaplan et al., 1991). In a different vein, Robertson (1990) found that degree of neglect was correlated with the discrepancy between forward and backward digit span, a parameter intended to capture attentional functioning. Robertson and Frasca (1992) have also reported that visual neglect may deteriorate when a non-visual secondary task (counting backwards in threes from 100) is given in addition to a simple basic visual detection task. This again emphasizes the importance of basic attentional processes in determining the severity of visual neglect.

Overall, these findings indicate that neglect is not a unitary phenomenon. Consequently, individual variation in performance may be, at least in part, due to the fact that standard tests map, to various degrees, different mechanisms responsible for asymmetrical performance. A logical analysis of the different requirements underlying those tasks indicates a number of relevant features.

Cancellation and drawing tasks heavily depend both upon a perceptual and a complex motor programme. In order to correctly perform the task, the subject has to explore the visual stimulus which typically extends across the vertical meridian, and make a number of movements (cross out or draw) both in the space contralateral and ipsilateral to the damaged hemisphere. In contrast, there are other tests which put emphasis on the overall perceptual judgment of the stimulus while requiring a minimal motor output. The line bisection test and the Wundt-Jastrow area illusion test (Massironi et al., 1988) seem to fall into this category. In the Wundt-Jastrow area illusion test the observer is required to judge which one of two fans placed one above the other is longer. Response can be given either verbally or by pointing. The same also appears true in the line bisection test; again, the observer has to make a single overall evaluation of line length. All these tests are characterized by the fact that only target stimuli are presented; however, there are versions of the cancellation tests in which a variable number of stimulus distractors are interspersed with the stimulus targets. As stated above, the comparison of tasks with and without stimulus distractors may be informative in demonstrating that some forms of asymmetrical performance may be detected only when a selective attentional load is placed on the task. ${ }^{1}$ In most studies, neglect for personal space has not been measured. However, a recent diagnostic procedure proved to be effective in identifying personal neglect as dissociated from extrapersonal neglect (Zoccolotti and Judica, 1991).

It seemed important to empirically test whether differences between tasks convincingly rely on different processes or can be considered variations of a common operation which may be tapped at different degrees of complexity.

' It may be noted that, in principle, a selective attention load can be applied to any type of task; however, to date, in standard testing only cancellation tests have been used with a systematic manipulation of stimulus distractors. Further work is needed to determine whether a selective attention load worsens performance on tests of perceptual or personal neglect. 
The present study investigates this question of left unilateral spatial neglect in a large population of right-braindamaged patients collected from various European teams and administered a variety of different tasks involving several modalities of spatial exploration with different perceptual, motor, attentional and personal or extrapersonal space dimensions. In light of previous results, we predicted a relatively high degree of communality among tests; namely, the presence of patients displaying an asymmetrical performance on all or none of the tests used. However, we also expected to find a number of patients with a more diversified performance profile. The analysis of these cases and, more generally, of the degree of similarity among tests (by means of a cluster analysis) might be informative as to whether individual variability may be test specific or may be accounted for on the basis of the logical distinctions briefly outlined above.

\section{METHODS}

\section{Sample}

One hundred and twenty-one right-brain-damaged patients with unilateral neglect were identified from seven laboratories (Bonn, Germany; Cambridge, UK; Dusseldorf, Germany; Edinburgh, UK; Oxford, UK; Paris, France; Rome, Italy). These patients showed signs of neglect in at least one of the eight tests used (see below).

There was a prevalence of patients with cerebral vascular accidents $(n=105,86.8 \%)$; fewer patients sustained a head injury $(n=8,6.6 \%)$, a neoplasia $(n=1,0.8 \%)$ or other kinds of pathology $(n=7,5.8 \%)$. Time from onset of disease presented a highly asymmetrical distribution (skewness $=3.91$ ): while most patients were seen within 1 year, a number of patients were examined several years after the onset of their pathology. Overall, median time was 6 months (range 1-312).

On average patients were 59.0 years old (S.D. $=14.5)$. Mean number of years of schooling was 9.0 (S.D. $=7.8$ ). There was a slightly larger percentage of males $(58.7 \%$ males and $41.3 \%$ females).

\section{Tests}

From the behavioural inattention test (BIT; Wilson et al., 1987a,b), only the six conventional subtests were used.

Line crossing. This visual search test requires the patient to detect and cross out all the target lines on the page. The examiner demonstrates the required response by crossing out two of the four lines located in the central column, and then instructs the subject to cross out all the lines he or she can see on the page. The four central lines are not scored. The maximum score is 36 .

Cut-off score: a patient is considered hemi-inattentive when the score is equal to or smaller than 34 . The cut-off score in all the six subtests of BIT was based on the worst performance of a normal control subject.

Letter cancellation. This paper and pencil test requires the patient to scan, locate and cross out designated targets from a background of distractor letters. The letter cancellation task consists of five rows of 34 upper-case letters presented on a rectangular page (297 by $210 \mathrm{~mm})$. The 40 ( $24 \%$ ) chosen targets ("E" and " $R$ ") are positioned so that an equal number appear on each side of the page. Each letter is $6 \mathrm{~mm}$ high and they are positioned close together ( $2 \mathrm{~mm}$ spacing). The maximum score is 40 and the scoring template allows the scorer to divide the total array into four columns, two on the left and two on the right. The total number of omitted target letters is calculated and the location of the omissions is noted. Cut-off score: equal to or less than 32 .

Star cancellation. The star cancellation test consists of a random array of verbal and non-verbal stimuli. The stimuli, 52 large stars $(14 \mathrm{~mm}), 13$ randomly positioned letters and 19 short (three-four letter) words, are interspersed with 56 smaller stars $(8 \mathrm{~mm})$ which comprise the target stimuli. The patient is instructed to cancel all the small stars. Two examples of the small stars are pointed out and cancellation of two central stars is demonstrated. As in the previous test, the test sheet can be subdivided into columns to calculate the number and location of errors. The maximum score is 54. Cut-off score: equal to or less than 51 .

Figure and shape copying. The patient was instructed to copy three separate simple drawings from the left side of the page. The three drawings, a four-pointed star, a cube and a daisy, are arranged vertically and are clearly indicated to the patient. The second part of this test requires the patient to copy a group of three geometric shapes presented on a separate stimulus sheet. Unlike the previous items, the contents of the page are not pointed out to the patient. Scoring is based on the completeness of the respective drawing. The presence of neglect is defined as an omission or gross distortion of any major contralesional component of the drawing. The maximum score is $4 .{ }^{2}$ Cutoff score: equal to or less than 3 .

Line bisection. This task requires the patient to estimate and indicate the midpoint of a horizontal line. Each patient is presented with three horizontal, 8 -inch $(204 \mathrm{~mm})$ black lines ( $1 \mathrm{~mm}$ thick) displayed in a staircase fashion across the page. The extent of each line is clearly pointed out to the patient who is then instructed to mark the centre. The

${ }^{2}$ As part of this European research project, a new more complex scoring procedure was developed for the figure- and shape-copying test and for representational drawing test; based on the new scoring, the maximum score is 24 and 18 for these two tests, respectively (Halligan et al., 1991b). 
test is scored by measuring deviations from the true midpoint.

Deviations to the left are scored as negative, to the right as positive. The deviation score is calculated using the normative data obtained from the age-matched controls. Using data from the control sample scores, values between 0 and $3(+/-)$ are assigned to the patient's performance for each of the three lines used. The maximum score is 9 . Cut-off score: equal to or less than 7 .

Representational drawing. The patient is instructed to draw pictures of a clock face, together with the numbers and a setting of the hands; a man or a woman; and a simple outline drawing of a butterfly. The task is designed to assess the patient's visual imagery independent of direct sensory input. The scoring is similar to that of the copying tasks, where neglect is defined as the omission or gross distortion of any major contralesional component of the drawing. The maximum score is 3. Cut-off score: equal to or less than 2 .

An additional two tests of neglect were employed.

Wundt-Jastrow area illusion test (Massironi et al., 1988). Two circular fans of identical shape and size are presented. As an effect of their relative position in space one appears longer. Ten sizes (ranging from $6^{\circ}$ to $58^{\circ}$ ), two orientations (upward-downward convexity) and two directions (leftward-rightward) are used for a total of 40 trials. The subject's task is to indicate which fan is larger. Responses are classified into two categories: "expected responses" are those consistent with the illusory effect in normals; "unexpected responses" are those in the opposite direction. Massironi et al. (1988) observed that right- brain-damaged patients with neglect made a considerable number of unexpected responses when the two fans were oriented in a leftward manner irrespective of stimulus size and orientation; in contrast, both right-brain-damaged patients with and without neglect and controls made very few unexpected responses for stimuli pointing toward the right. Therefore, the raw score is the number of unexpected responses when the two fans are oriented toward the left or the right. Cut-off score: the patient is considered hemi-inattentive when a difference between unexpected responses given for left-oriented minus right-oriented stimuli is equal to or greater than 2 (Massironi et al., 1988).

\section{Semi-structured scale for the functional evaluation of personal neglect (Zoccolotti and Judica, 1990, 1991).} The patient is presented with three objects, one at a time (comb, razor (for man)/powder (for woman), eyeglasses) and he/she is asked: "Show me how you use___?". The exploratory behaviours of the patients are classified into four performance levels. In particular, the patient's behaviour is considered normal where no systematic asymmetries in exploration are found (score 0 ). At a second level, the patient may complete the task exploring the entire space or only very slight asymmetries may be present (score 1); what characterizes this level is the presence of uncertainty and slowness in arriving in the space opposite that of the lesion. At a third level, clear omissions in the space opposite that of the lesion are present (score 2). At the final level, the patient is only able to explore a very reduced portion of the space (relative to that ipsilateral to the lesion) (score 3).

One separate evaluation for each of the three objects (comb, eyeglasses and razor/powder) is made. Thus, on the whole, the scale produces a total of three evaluations of

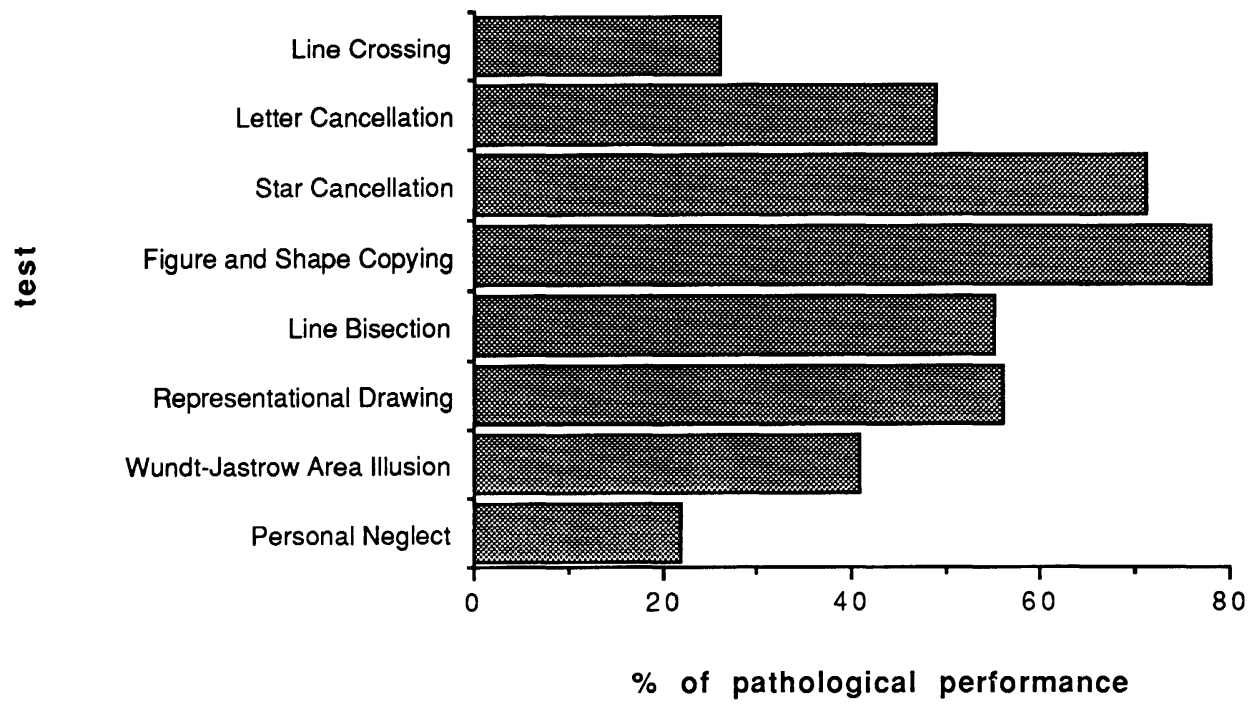

FIG. 1. Percentage of patients below the cut-off point of normal performance in each of the tests used. 
TABLE I. Medians, ranges and maximum possible scores for the eight tests considered. For the first six tests (BIT battery) the score is the number of correct responses. For the Wundt-Jastrow area illusion test, the score represents the number of responses inconsistent with the normal illusory effect when the two fans appear pointing to the left. For the semi-structured scale of personal neglect, the score is the sum of qualitative judgments of patient's behaviour in three situations (greater score indicates greater asymmetry of behaviour)

\begin{tabular}{lcccc}
\hline Test & Median & Range & $\begin{array}{c}\text { Possible } \\
\text { score }\end{array}$ & $\begin{array}{c}\text { Cut- } \\
\text { off }\end{array}$ \\
\cline { 2 - 5 } & 36 & $6-36$ & $0-36$ & 34 \\
Line crossing & 33 & $0-40$ & $0-40$ & 32 \\
Letter cancellation & 48 & $4-54$ & $0-54$ & 51 \\
Star cancellation & 2 & $0-4$ & $0-4$ & 3 \\
Figure copying & 7 & $0-9$ & $0-9$ & 7 \\
Line bisection & 2 & $0-3$ & $0-3$ & 2 \\
Representational drawing & 1 & $0-20$ & $0-20$ & $\mathrm{~L}-\mathrm{R}>2$ \\
Wundt-Jastrow area & & & & \\
$\quad$ illusion test & 0 & $0-9$ & $0-9$ & 2 \\
$\quad \begin{array}{l}\text { Semi-structured scale } \\
\quad \text { of personal neglect }\end{array}$ & & & & \\
\hline
\end{tabular}

patients' explorative behaviour with a total score between 0 and 9. Cut-off score: the patient is considered hemi-inattentive when he/she receives a score greater than or equal to 2 (Zoccolotti et al., 1992).

\section{RESULTS}

The proportion of patients falling below the cut-off point for pathological performance in the various tests is shown in Fig. 1. Considerable variability is observed across tests in their sensitivity to detect pathological performance. Less than $30 \%$ of patients were pathological on the line crossing test; an even lower percentage was observed in the case of the semi-structured scale of the functional evaluation of personal neglect. On the star cancellation and the figure-copying tests, more than $70 \%$ of the patients performed below the cut-off point.

Medians (and ranges) for the eight tests are presented in Table I. The relationship between the performance in the
TABLE II. Spearman correlations of the various tests with time from onset of CVA, age and number of years of schooling

\begin{tabular}{lrrc}
\hline Test & $\begin{array}{c}\text { Time from } \\
\text { onset of CVA }\end{array}$ & Age & $\begin{array}{c}\text { Years } \\
\text { of schooling }\end{array}$ \\
\hline Line crossing & -0.15 & -0.08 & 0.02 \\
Letter cancellation & -0.14 & -0.08 & 0.04 \\
Star cancellation & -0.06 & -0.05 & $-0.23^{\star}$ \\
Figure copying & 0.02 & -0.02 & -0.02 \\
Line bisection & 0.09 & -0.00 & -0.07 \\
Representational drawing & -0.16 & -0.02 & 0.14 \\
Wundt-Jastrow area & -0.10 & 0.11 & 0.03 \\
$\quad$ illusion test & & & \\
Semi-structured scale & -0.17 & $0.19^{*}$ & 0.14 \\
$\quad$ of personal neglect & & & \\
\hline${ }^{*} p<0.05$, two-tailed test. & & &
\end{tabular}

eight tests and the time from onset of disease (for this parameter, only patients with cerebrovascular aetiology were considered), age and education is presented in Table II. In general, for all tests, performance of patients was not correlated with the time interval from cerebral injury. Overall, these results are consistent with the idea that, within a non-acute patient population, performance in exploratory tests is essentially stable over time in patients with the neglect syndrome (Zarit and Kahn, 1974; Zoccolotti et al., 1989). Also in the case of age, no relationship was found for most tests. For the semi-structured scale for the functional evaluation of personal neglect, performance tended to be worse with increasing age (see Table II). Finally, educational level was associated with performance only on the star cancellation (see Table II).

\section{Relationship among tests}

The correlation matrix (using Spearman's correlation) among the eight tests considered is presented in Table III. Considering the number of comparisons, a 0.001 (onetailed test) level of significance was chosen. In general, with the exception of the semi-structured scale of personal neglect, all tests gave rather consistent results. Thus, they were all intercorrelated (with the exception of the line

TABLE III. Spearman correlations among the eight tests considered

\begin{tabular}{|c|c|c|c|c|c|c|c|c|}
\hline Test & $\begin{array}{l}\text { Line } \\
\text { crossing }\end{array}$ & $\begin{array}{c}\text { Letter } \\
\text { cancellation }\end{array}$ & $\begin{array}{c}\text { Star } \\
\text { cancellation }\end{array}$ & $\begin{array}{l}\text { Figure } \\
\text { copying }\end{array}$ & $\begin{array}{l}\text { Line } \\
\text { bisection }\end{array}$ & $\begin{array}{l}\text { Representational } \\
\text { drawing }\end{array}$ & $\begin{array}{l}\text { Wundt- } \\
\text { Jastrow }\end{array}$ & $\begin{array}{l}\text { Personal } \\
\text { neglect }\end{array}$ \\
\hline Line crossing & - & $0.56^{*}$ & $0.48^{*}$ & $0.47^{\star}$ & $0.39^{*}$ & 0.25 & $-0.37^{\star}$ & -0.20 \\
\hline Letter cancellation & & - & $0.65^{\star}$ & $0.48^{*}$ & $0.53^{*}$ & $0.33^{\star}$ & $-0.48^{\star}$ & -0.25 \\
\hline Star cancellation & & & - & $0.54^{*}$ & $0.65^{\star}$ & $0.35^{*}$ & $-0.62^{\star}$ & $-0.38^{*}$ \\
\hline Figure copying & & & & - & $0.48^{*}$ & $0.48^{\star}$ & $-0.55^{\star}$ & -0.16 \\
\hline Line bisection & & & & & 一 & $0.31^{*}$ & $-0.54^{\star}$ & $-0.34^{*}$ \\
\hline Representational drawing & & & & & & - & $-0.42^{\star}$ & -0.003 \\
\hline $\begin{array}{l}\text { Wundt-Jastrow area } \\
\text { illusion test }\end{array}$ & & & & & & & - & $-0.37^{\star}$ \\
\hline $\begin{array}{c}\text { Semi-structured scale } \\
\text { of personal neglect }\end{array}$ & & & & & & & & - \\
\hline
\end{tabular}

${ }^{\star} p<0.001$, one-tailed test. 
crossing and representational drawing tests). The semistructured scale of personal neglect was correlated with the star cancellation, the line bisection test and the WundtJastrow area illusion test but not with the other four tests of neglect.

The relationship among the various tests administered was also examined by means of a cluster analysis based on the average linkage method (Aldefender and Blashfield, 1984). In the case of ordinal data, a Minkowski distance, such as the squared Euclidean distance $\left(\Sigma\left(x_{\mathrm{i}}-y_{\mathrm{i}}\right)^{2}\right)$, is commonly taken as an index of similarity among variables (e.g. Rizzi, 1985).

A dendrogram describing the relationship between the eight tests is presented in Fig. 2. To examine the number of clusters, the progression of the agglomeration coefficients (indicating the level of distance in the linkage of two groups) is evaluated: a relatively large increase in the agglomeration coefficient indicates that the two groups of observations are not similar to each other. A clear differentiation is present between the semi-structured scale for the functional evaluation of personal neglect and all other tests. This finding is consistent with a distinction between tasks involving personal and extrapersonal space.

A further examination of the dendrogram referring to the various extrapersonal tests does not point to the clear emergence of additional cluster segregations. Rather, there appears to be a "chaining" effect, such that different tests seem to enter the dendrogram in a meaningful way. Thus, an examination of the degree of similarity among the various extrapersonal tests suggests a number of additional observations.

The star cancellation and letter cancellation tests enter the cluster first. These two tests clearly show a "face" similarity in the response mechanisms and in the cognitive structure of the test, which will be discussed below. After a relatively large increase in the agglomeration coefficient, two tests enter close to each other in the agglomeration tree (line bisection and Wundt-Jastrow area illusion tests). The

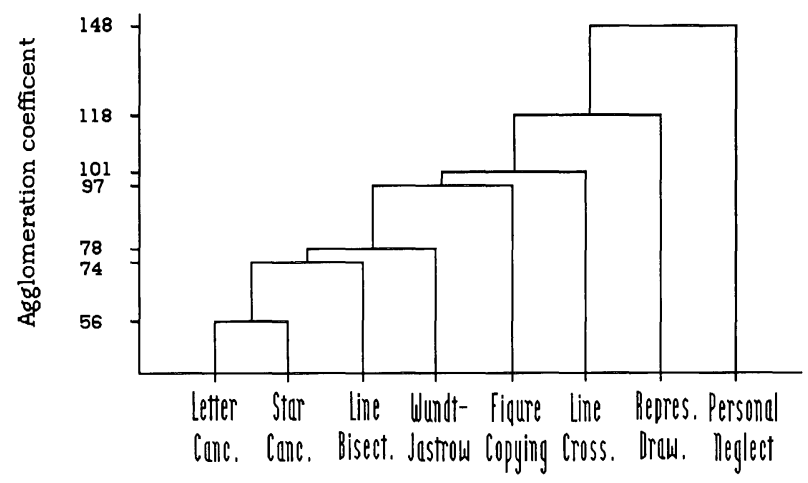

FIG. 2. Dendrogram of the eight tests of neglect used. The ordinate indicates squared Euclidian distance among variables taken as an index of similarity. similarity between these two tests has been described in the Introduction and will be further discussed below. After a further increase in the agglomeration coefficient, two tests enter the dendrogram (line crossing and figure and shape copying). These two tests require the patient to systematically screen the complex stimulus and to organize a fine and sequential motor response. It should be added that the line crossing does not require, as in the case of letter and star cancellation tasks, any selection of the target stimuli among distractors. Representational drawing is characterized by a considerable increase in the agglomeration coefficient and must be considered to be relatively independent of the other tests.

This general pattern of results was replicated by the use of several different cluster analyses (complete linkage method, single linkage method, Ward method), with only minor differences. Overall, this finding points to the relative stability of the solution obtained (Aldefender and Blashfield, 1984). Furthermore, considering the large variability of the sample characteristics, it was decided to replicate the analysis eliminating all patients who could possibly have bilateral or diffuse lesions. Ten patients with either clinical signs of dementia or head injury were eliminated from the sample. The new set of cluster analyses performed on 111 patients reproduced the same pattern, with only marginal differences.

\section{Analysis of individual cases}

To further support the distinctions proposed in the Introduction, we looked at individual cases showing the presence of a selective neglect impairment. More specifically, we hypothesized that there might be individual patients displaying only personal neglect or neglect only on tests requiring an overall perceptual evaluation, with a minimal motor component. Another dissociation could be expected between tests requiring a fine and sequential motor output with an additional requirement to select the target stimuli from distractors (letter and star cancellation) and tests demanding similar motor responses without involving any selective component. We decided to consider an individual case as a patient with perceptual neglect if his performance was pathological compared to controls on both the line bisection and the Wundt-Jastrow area illusion tests; at the same time, no pathological performance should have been present on any of the other six neglect tests. A similar procedure was used to define the presence of patients with neglect in tasks involving selective attention (pathological performance in both the star cancellation and letter cancellation tests only). The presence of personal neglect was defined as the selective failure in only the semi-structured scale for the functional evaluation of personal neglect. While most patients showed a mixed or complete pattern of pathological performance, some of the predictions of dissociation were supported. In 
particular, three patients showed a selective deficit only on the scale of personal neglect; three additional patients showed a deficit on both the star cancellation and letter cancellation tests but were flawless on every other test. In contrast with our expectations, we did not find any patients displaying perceptual neglect only.

\section{DISCUSSION}

Consistent with previous research, we observed that sensitivity to detect pathological performance in neglect tests was task dependent (e.g. Horner et al., 1989). For tasks mapping extrapersonal space, scores above the cut-off varied from $25 \%$ to $75 \%$. Confirming previous research (Rapcsak et al., 1989; Kaplan et al., 1991), relatively high estimates of neglect were present in the case of those tests which emphasized selective attention.

The main result of the cluster analysis is a clear segregation of measures of personal vs extrapersonal neglect. This finding was independent of the method used to identify the clusters. Furthermore, even if personal neglect was relatively more infrequent than extrapersonal neglect, it seems meaningful that there were patients showing personal without extrapersonal neglect and vice versa. Altogether these findings support the hypothesis of separate coding of inner and outer space (e.g. Rizzolatti and Camarda, 1987). From a clinical point of view this dissociation stresses the importance of directly and separately testing for personal neglect in a battery which aims to identify the possible manifestations of neglect. While a similar dissociation has been previously described (Zoccolotti and Judica, 1991), the larger sample of neglect patients used in the present research gives the first estimate of its relative incidence in a population of chronic patients. About $20 \%$ of the patients with right hemispheric lesions showing neglect presented some degree of personal neglect either in isolation or in addition to extrapersonal neglect.

In the other large cluster, which we labelled extrapersonal neglect, the seven tests, although strongly linked with each other, showed a pattern of similarities which can be tentatively interpreted in an understandable way. The fact that the different tasks do not segregate to form separate clusters is probably a consequence of the kind of population studied. Such a large sample of patients unselected for aetiology, age, education, distance from the ictus and usually with large size lesions makes it unlikely that clearcut isolated impairments would emerge, such as the ones previously reported in the literature. Also, it should be noted that dissociations are often based on the experimental manipulation of a given task. For example, it was shown that individual patients may respond differently in a cancellation task depending on whether or not the visual input is inverted by means of specifically devised mirrors (Tegner and Levander, 1991). Standard tests may be somewhat less pure in detecting the role of specific processes (e.g. perceptual vs motor). However, in turn one might think that fractionation of performance in standard tests may be more readily useful in a clinical setting. With this in mind, the present data still show a non-random pattern of task similarity.

The first two tasks which group together are the star cancellation and letter cancellation tests. These two tests share the task of sequentially analysing the target stimuli. Furthermore, in contrast to all the other tests, they are also characterized by the presence of a (large) number of stimulus distractors. It has been suggested that this requirement indicates the role of selective attention in the performance of these types of tests (Rapcsak et al., 1989). Analysis of individual cases indicated that three patients showed asymmetrical performance in both these tests but were within the range of normal performance in all the other tests used, thus confirming the qualitative peculiarity of this type of task. More generally, these observations are consistent with the idea that differential sensitivity in detecting pathological performance is not random but depends on the type of processes involved in the task. Then, these two tasks grouped with the line bisection and the Wundt-Jastrow area illusion tests. We have suggested that both of these tests rely more on the evaluation of perceptual input; in contrast, in both cases, the motor response is very simple. Furthermore, both tasks require that the patient examine the stimulus as a whole instead of attending to its separate components. The similarity between the two tasks can be tentatively considered as consistent with the idea that they represent an indicator of "perceptual neglect". However, a note of caution is necessary here, since no patient with a selective deficit in these two tests only was found. Perhaps areas contributing to perceptual and motor processes in exploratory tasks are closely contiguous in the human brain, thus reducing the likelihood of detecting dissociated cases. Alternatively, one might think that even the minimal motor requirements required in these two tests are sufficient to produce performances that cannot be distinguished from tests with a clear motor component (such as drawing and cancellation tests).

Another relevant finding of the present study refers to the low correlations between performance in the various tests and both the time interval from the onset of disease and age. These data confirm previous observations (Zarit and Kahn, 1974; Zoccolotti et al., 1989) on extrapersonal tests of neglect and extend them to the issue of personal neglect, for which no cross-sectional developmental analyses have yet been reported. More generally, they clearly indicate the relative stability of neglect and its independence from age in a population of chronic braindamaged patients. 
Overall, these observations have important clinical implications. First, the finding that neglect may be long lasting indicates the need for a rehabilitative intervention when it persists beyond the acute phase. Second, the differential sensitivity and the qualitative difference among tests points to the need for using a relatively large and diversified set of tasks in order to maximize the possibility of identifying neglect disorders. The simple use of one task will most likely result in the missing of a consistent number of pathological cases. A battery such as the present one is at the same time sufficiently easy to administer and capable of identifying a large number of neglect patients.

\section{Acknowledgements}

This research was carried out as part of the European Research Co-operation on "The Evaluation of the Efficacy of Technology in the Assessment and Rehabilitation of Brain-damaged Patients". The authors would like to thank Mrs S. Balmas, Mrs C. Razzano, Dr P. Pradat-Diehl, Mrs I. Bureca and Mr A. Matano for their help in collecting the data, and Dr G. Gainotti and A. Basso for critical reading of the manuscript.

\section{REFERENCES}

Aldefender M and Blashfield R (1984) Cluster Analyses. Sage Publications, Beverly Hills.

Bisiach E and Vallar G (1988) Hemineglect in humans. In: Handbook of Neuropsychology, Vol. 1 (Eds F Boller and J Grafman), pp. 195-222. Elsevier, Amsterdam.

Bisiach E, Perani D, Vallar G and Berti A (1986) Unilateral neglect: personal and extrapersonal. Neuropsychologia, 24, 759-767.

Brain WR (1941) Visual disorientation with special reference to lesions of the right hemisphere. Brain, 64, 244-272.

De Renzi E (1982) Disorders of Space Exploration and Cognition. Wiley, London.

De Renzi, Colombo A, Faglioni P and Gibertoni M (1982) Conjugate gaze paresis in stroke patients with unilateral damage. Archives of Neurology, 39, 482-486.

Gainotti G (1968) Les manifestations de negligence et d'inattention pour l'hemispace. Cortex, 4, 64-91.

Guariglia C and Antonucci G (1992) Dissociation in man of extrapersonal and personal space representation. Neuropsychologia, 30, 1001-1009.

Halligan P and Marshall J (1991) Left neglect for near but not far space in man. Nature, 350, 498-500.

Halligan PW, Marshall JC and Wade, DT (1989) Visuospatial neglect; underlying factors and test sensitivity. Lancet, 2, 908-911.

Halligan P, Cockburn J and Wilson B (1991a) The behavioural assessment of visual neglect. Neuropsychological Rehabilitation, 1, 5-32.

Halligan P, Robertson I, Pizzamiglio L, Homberg V, Weber E and Bergego C (1991b) Visual neglect after right hemisphere damage. Neuropsychological Rehabilitation, 1, 281-301.

Heilman KM, Valenstein E and Watson RT (1985) The neglect syndrome. In: Handbook of Clinical Neurology (Eds PJ Vinken, GW Bruyn and HL Klawans). Elsevier, Amsterdam.
Hier DB, Mondlock J and Caplan LR (1983) Behavioural abnormalities after right hemispheric stroke. Neurology, 33, 337-344.

Horner J, Massey W, Woodruff W, Chase K and Dawson D (1989) Task-dependent neglect: Computer tomography size and locus correlations. Journal of Neurological Rehabilitation, 3, 713.

Jeannerod, M (Ed) (1987) Neurophysiological and Neurophychological Aspects of Spatial Neglect. North-Holland, Amsterdam.

Kaplan RF, Verfaellie M, Meadows M, Caplan LR, Pessin MS and De Witt D (1991) Changing attentional demands in left hemispatial neglect. Archives of Neurology, 48, 1263-1266.

Massironi M, Antonucci G, Pizzamiglio L, Vitale MV and Zoccolotti P (1988) The Wundt-Jastrow illusion in the study of spatial hemi-inattention. Neuropsychologia, 26, 161-166.

Pizzamiglio L, Cappa S, Vallar G, Zoccolotti P, Bottini G, Ciurli P, Guariglia C and Antonucci G (1989) Visual neglect for far and near extra-personal space in humans Cortex, 25, 471-477.

Rapcsak SZ, Varfaellie M, Fleet WS and Heilman KM (1989) Selective attention in hemispatial neglect. Archives of Neurology, 46, 178-182, 1989.

Rizzi A (1985) Analisi dei dati. NIS, Roma.

Rizzolatti G and Camarda R (1987) Neural circuits for spatial attention and unilateral neglect. In: Neurophysiological and Neuropsychological Aspects of Spatial Neglect (Ed. M Jeannerod), pp. 289-313. Elsevier, Amsterdam.

Robertson I (1990) Digit span and visual neglect: a puzzling relationship. Neuropsychologia, 28, 217-222.

Robertson IH and Frasca R (1992) Attentional load and visual neglect. International Journal of Neuroscience, 62, 45-56.

Robertson I and Marshall J (1992) Unilateral Neglect. Clinical and Experimental Studies. Erlbaum, Hillsdale.

Smith DL, Akhtar AJ and Garraway WM (1983) Proprioception and spatial neglect after stroke. Age and Ageing, 12, 63-69.

Tegner R and Levander M (1991) Through a looking glass. A new technique to demonstrate directional hypokinesia in unilateral neglect. Brain, 114, 1943-1951.

Wilson B, Cockburn J and Halligan P (1987a) Development of a behavioral test of visuospatial neglect. Archives of Physical Medicine and Rehabilitation, 68, 98-102.

Wilson B, Cockburn J and and Halligan P (1987b) Behavioral Inattention Test. Thames Valley Test Company, Titchfield, Hants.

Zarit SH and Kahn RL (1974) Impairment and adaptation in chronic disabilities: spatial inattention. Journal of Nervous and Mental Disease, 159, 63-72.

Zoccolotti P and Judica A (1990) Validazione di una scala semistrutturata per la valutazione funzionale del disturbo eminattentivo. Archivio di Psicologia, Neurologia e Psichiatria, 51, 364-385.

Zoccolotti P and Judica A (1991) Functional evaluation of hemineglect by means of a semi-structured scale: personal extrapersonal differentiation. Neuropsychological Rehabilitation, 1, 33-44.

Zoccolotti P, Antonucci G, Judica A, Montenero P, Pizzamiglio $\mathrm{L}$ and Razzano C (1989) Incidence and evolution of the hemineglect disorder in chronic patients with unilateral right brain damage. International Journal of Neuroscience, 47, 209-216.

Zoccolotti P, Antonucci G and Judica A (1992) Psychometric characteristics of two semi-structured scales for the functional evaluation of hemi-inattention in extra-personal and personal space. Neuropsychological Rehabilitation, 2, 179-192. 


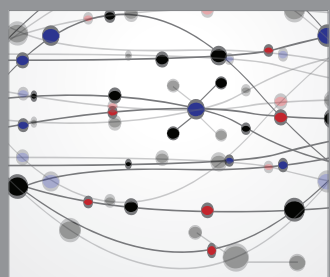

The Scientific World Journal
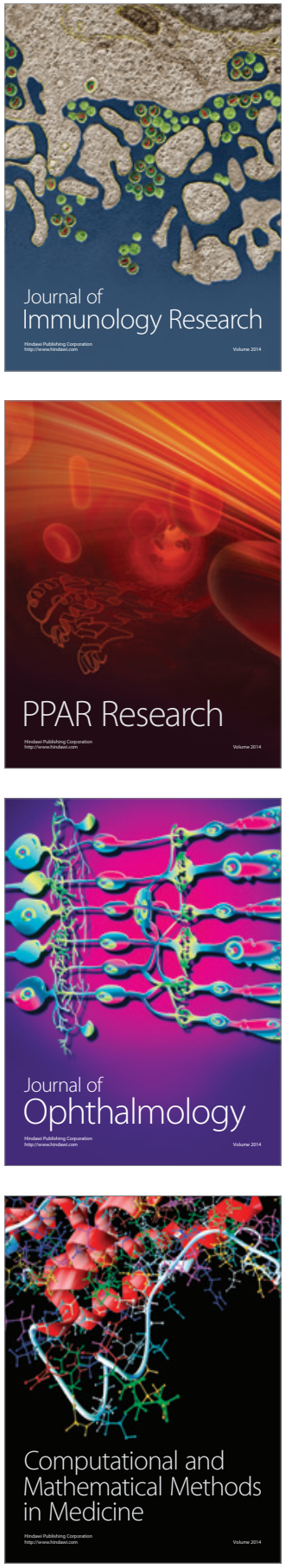

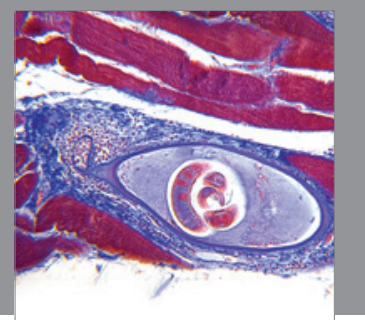

Gastroenterology

Research and Practice
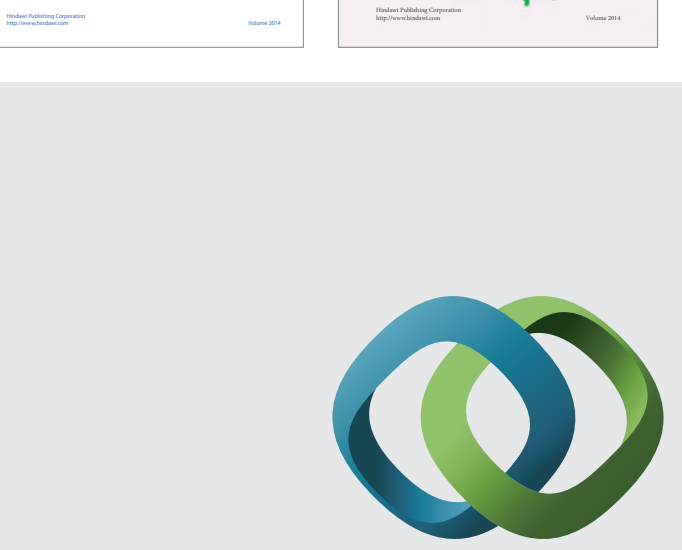

\section{Hindawi}

Submit your manuscripts at

http://www.hindawi.com
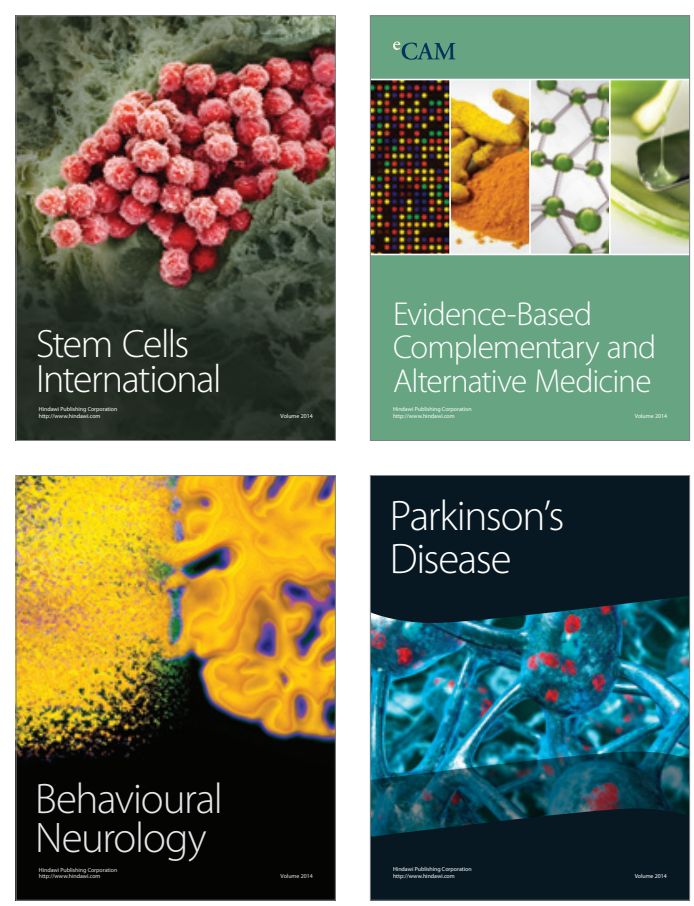

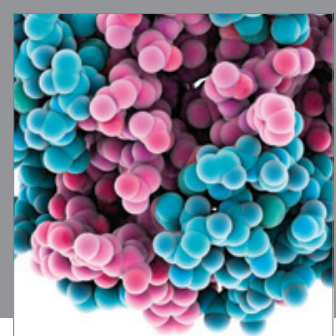

Journal of
Diabetes Research

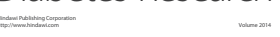

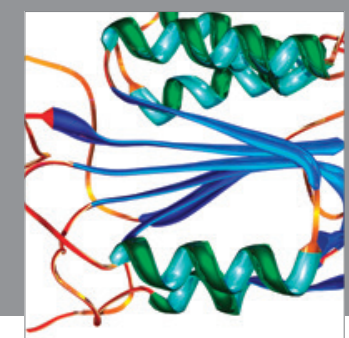

Disease Markers
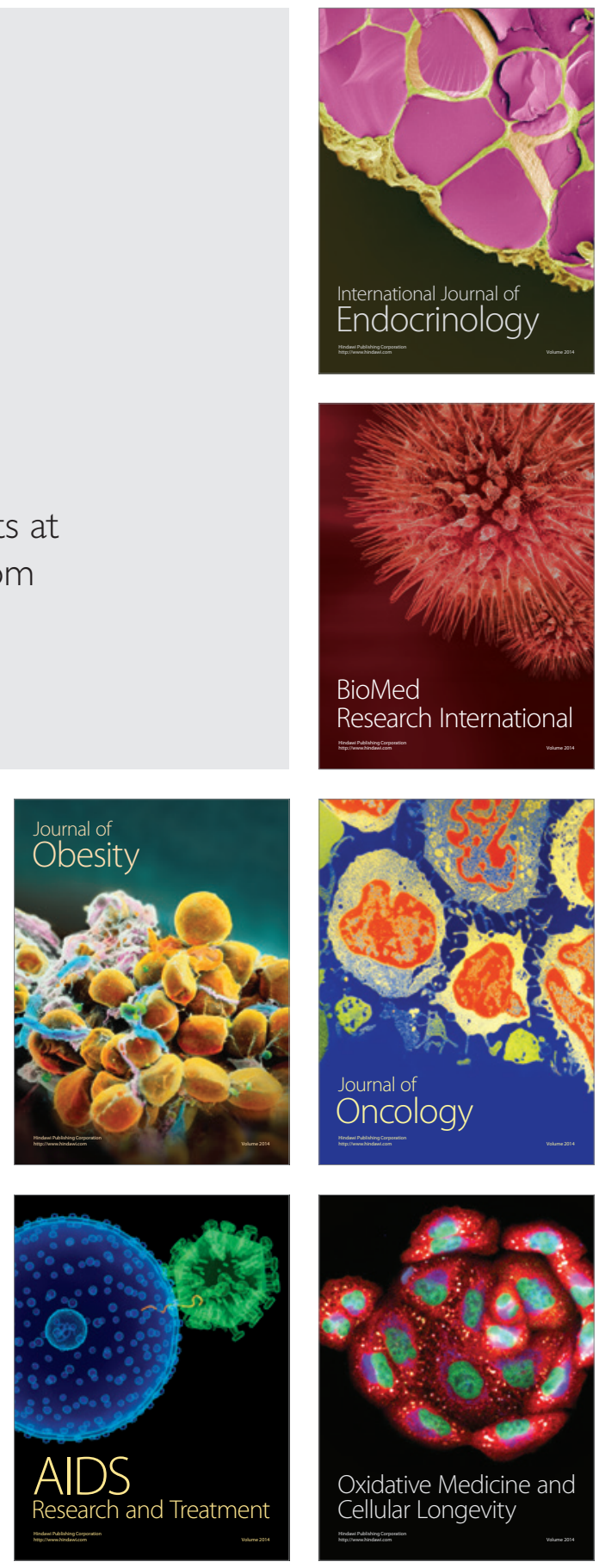